Program for Promoting Social Science Research

Aimed at Solutions of Near-Future Problems

Design of Interfirm Network to Achieve Sustainable Economic Growth

Working Paper Series No.11

\title{
Power Laws in Firm Productivity
}

\author{
Takayuki Mizuno \\ Atushi Ishikawa \\ Shouji Fujimoto \\ and \\ Tsutomu Watanabe
}

Novem ber 15, 2011

Research Center for Interfirm Network

Institute of Economic Research, Hitotsubashi University

Naka 2-1, Kunitachi-city, Tokyo 186-8603, JAPAN

Tel: $+81-42-580-9145$

E-mail: hit-tdb-sec@ier.hit-u.ac.jp

http://www.ier.hit-u.ac.jp/ifn/ 


\title{
Power Laws in Firm Productivity
}

\author{
Takayuki Mizuno ${ }^{1,2}$, Atushi Ishikawa ${ }^{3}$, Shouji Fujimoto ${ }^{2,3}$, \\ Tsutomu WatANABE ${ }^{2,4}$ \\ ${ }^{1}$ Division of Information Engineering, Faculty of Engineering, Information and \\ Systems, University of Tsukuba, 1-1-1 Tennodai, Tsukuba, Ibaraki 305-8573, Japan \\ ${ }^{2}$ Canon Institute for Global Studies, 1-5-1 Marunouchi, Chiyoda-Ku, Tokyo \\ 100-6511, Japan. \\ ${ }^{3}$ Department of Informatics and Business, Faculty of Business Administration and \\ Information Science, Kanazawa Gakuin University, 10 Sue, Kanazawa, Ishikawa \\ 920-1392, Japan \\ ${ }^{4}$ Graduate School of Economics, University of Tokyo, 7-3-1 Hongo, Bunkyo-ku, \\ Tokyo 113-0033, Japan
}

\begin{abstract}
We estimate firm productivity for about 3.2 million firms from 30 countries. We find that the distribution of firm productivity in each country, which is measured by total factor productivity (TFP), has a power law upper tail. However, the power law exponent of a TFP distribution in a country tends to be greater than that of a sales distribution in that country, indicating that the upper tail of a TFP distribution is less heavy compared to that of a sales distribution. We also find that the power law exponent of a TFP distribution tends to be greater than the power law exponents associated with the number of workers or tangible fixed assets. Given the idea that the sales of a firm is determined by the amount of various inputs employed by the firm (i.e., "production function" in the terminology of economics), these results suggest that the heavy tail of a sales distribution in a country comes not from the tail of a TFP distribution, but from the tail of the distribution of the number of workers or tangible fixed assets.
\end{abstract}

\section{$\S 1$. Introduction}

Ever since Pareto reported 120 years ago that the distribution of personal wealth follows a power law, both physicists and economists have investigated the distributions of a wide variety of size variables. ${ }^{1)}$ Some economists found in the 1950 s and 1960s that firm size distributions have fatter tails than log-normal distributions, ${ }^{2}{ }^{-4)}$ while physicists showed in the 1990s and later that the distributions of firm size variables, including firm sales, the number of workers, and tangible fixed assets, are characterized by power law tails. ${ }^{5}{ }^{-9}$ ) The mechanism behind firm growth was also investigated so as to learn more about where the power law tails of firm size distributions come from. ${ }^{10)-13)}$

More recently, researchers have shifted their focus to the relationship between firm sales, the number of workers, and tangible fixed assets. ${ }^{14-16)}$ It was shown that this relationship is not linear but nonlinear, $\left.{ }^{5}\right), 14$ ) and that there exists a scaling law for the relationship. ${ }^{14)}$ On the other hand, some economists studied this relationship based on the idea of "production function", ${ }^{17)-20)}$ in which the output of a firm is determined by the amount of inputs employed by the firm, including the number of workers, tangible fixed assets, and productivity. Among several alternative functional 
forms for production function proposed by economists, the most widely used one is a Cobb-Douglas production function, ${ }^{21)}$ which is given by $Y=A K^{\alpha} L^{\beta}$, where $Y$ is output, $L$ is the number of workers, $K$ is tangible fixed assets, and $A$ is productivity (more precisely, total factor productivity, or TFP). The exponents $\alpha$ and $\beta$ are constant parameters, which are usually estimated empirically.

Note that $Y, L$, and $K$ are reported by firms in their financial statements, so that they are all observable. However, $A$ is not observable, so that we need to estimate it. Recently, it was found using firm level data from major countries, including the U.S. and Japan, that the relationship between $Y, L$, and $K$ is well described by a CobbDouglas production function at least for mega firms (i.e. firms that belong to the tail part of firm size distributions). ${ }^{14), 17)}$ This suggests a method to estimate $A$ from the data; namely, we first estimate the exponents $\alpha$ and $\beta$ of a Cobb-Douglas production function using the data of $Y, L$, and $K$, and then compute $A$ by $A=Y /\left(K^{\alpha} L^{\beta}\right)$. In the present paper, we will estimate TFP in this way, and then look at the tail part of its distribution. Specifically, we are interested in whether the distribution of TFP is characterized by power law tails, as in the case of the distributions for $Y, L$, and $K$, and whether the tail of a TFP distribution is thicker or thinner than the tails associated with $Y, L$, and $K$.

The rest of the paper is organized as follows. In Sections 2 to 4, we estimate TFP for about 3.2 million firms from 30 countries, with the sample period of 2004 to 2008. The data we will use is from "Orbis," a database compiled by Bureau van Dajk Electronic Publishing. In Section 2, we give a brief review of the method proposed by Ishikawa et al. for estimating a Cobb- Douglas production function. In Section 3, we estimate two key parameters, $\alpha$ and $\beta$, of a Cobb-Douglas function and compare them across countries, as well as across industries. In Section 4, we show that the distribution of TFP is characterized by a power law tail, and that its power law exponent is greater than those for $Y, L$, and $K$ for most countries, as well as for most industries. In Section 5, we use value added instead of sales as a measure of output in estimating TFP. This is closer to the traditional treatment adopted by economists. We repeat the same exercise as in Sections 3 and 4 to confirm that the basic results remain unchanged. Section 6 concludes the paper.

\section{$\S 2$. Estimation of Cobb-Douglas production functions}

It is shown by previous studies that various firm size variables, including firm sales $(S)$, the number of workers $(L)$, and tangible fixed assets $(K)$, follow power law distributions in many countries. That is,

$$
\begin{array}{ccc}
P_{>}(S) \propto S^{-\mu_{S}} & \text { for } & S>S_{0} \\
P_{>}(L) \propto L^{-\mu_{L}} & \text { for } & L>L_{0} \\
P_{>}(K) \propto K^{-\mu_{K}} & \text { for } & K>K_{0}
\end{array}
$$

where $S_{0}, L_{0}$, and $K_{0}$ represent the size thresholds, and $\mu_{S}, \mu_{L}$, and $\mu_{K}$ are the power law exponents associated with $S, L$, and $K$. Ishikawa et al. found two statistical laws regarding the relationships between $S, L$, and $K .{ }^{14)}$ The first law is that the 
joint probability distributions of $S$ and $L, S$ and $K, L$ and $K$ are characterized by nonlinear functions of the form

$$
\begin{gathered}
P_{J}(L, S)=P_{J}\left(\left(\frac{S}{A_{L S}}\right)^{\frac{1}{\nu_{L S}}}, A_{L S} L^{\nu_{L S}}\right) \quad \text { for } \quad L>L_{0}, \\
P_{J}(S, K)=P_{J}\left(\left(\frac{K}{A_{S K}}\right)^{\frac{1}{\nu_{S K}}}, A_{S K} S^{\nu_{S K}}\right) \text { for } \quad S>S_{0}, \\
P_{J}(L, K)=P_{J}\left(\left(\frac{K}{A_{L K}}\right)^{\frac{1}{\nu_{L K}}}, A_{L K} L^{\nu_{L K}}\right) \text { for } L>L_{0},
\end{gathered}
$$

where the coefficients $A_{L S}, A_{S K}, A_{L K}$ and $\nu_{L S}, \nu_{S K}, \nu_{L K}$ are fixed numbers. The second law is that $S, L$, and $K$ satisfy Gibrat's law as

$$
\begin{gathered}
P\left(\frac{S}{A_{L S} L^{\nu_{L S}}} \mid L\right)=P\left(\frac{S}{A_{L S} L^{\nu_{L S}}}\right) \quad \text { for } \quad L>L_{0}, \\
P\left(\frac{K}{A_{S K} S^{\nu_{S K}}} \mid S\right)=P\left(\frac{K}{A_{S K} S^{\nu_{S K}}}\right) \text { for } \quad S>S_{0}, \\
P\left(\frac{K}{A_{L K} L^{\nu_{L K}}} \mid L\right)=P\left(\frac{K}{A_{L K} L^{\nu_{L K}}}\right) \quad \text { for } \quad L>L_{0} .
\end{gathered}
$$

From these two laws, Ishikawa et al. derives the following relational expression. ${ }^{14)}$

$$
S=A K^{\alpha} L^{\beta} \quad \text { for } \quad L>L_{0} \quad \text { and } \quad K>K_{0},
$$

where the coefficients $\alpha, \beta$ are fixed numbers, and $A$ is a stochastic variable which is independent of $K$ and $L$, so that $A$ satisfies

$$
P(A \mid K, L)=P(A) .
$$

In economics, Eq. (2.10) is known as a Cobb-Douglas production function, while the variable $A$ is referred to as total factor productivity, or TFP.

In estimating the coefficients $\alpha$ and $\beta$ in Eq. (2.10), we need pay attention to the issue of multicollinearity; namely, $K$ and $L$ are highly correlated so that a simple application of ordinary least squares does not work. To overcome this issue, Ishikawa et al. proposes to introduce new variables, $Z_{1}$ and $Z_{2}$, which are defined as

$$
\begin{aligned}
& \log Z_{1}=\frac{\log L}{\sigma_{\log L}}+\frac{\log K}{\sigma_{\log K}}, \\
& \log Z_{2}=\frac{\log L}{\sigma_{\log L}}-\frac{\log K}{\sigma_{\log K}},
\end{aligned}
$$

where $\sigma_{\log L}$ and $\sigma_{\log K}$ are the standard deviations of $\log K$ and $\log L$. Then Eq. (2.10) is transformed into

$$
S=A Z_{1}^{\theta_{1}} Z_{2}^{\theta_{2}} \quad \text { for } \quad L>L_{0} \text { and } K>K_{0},
$$




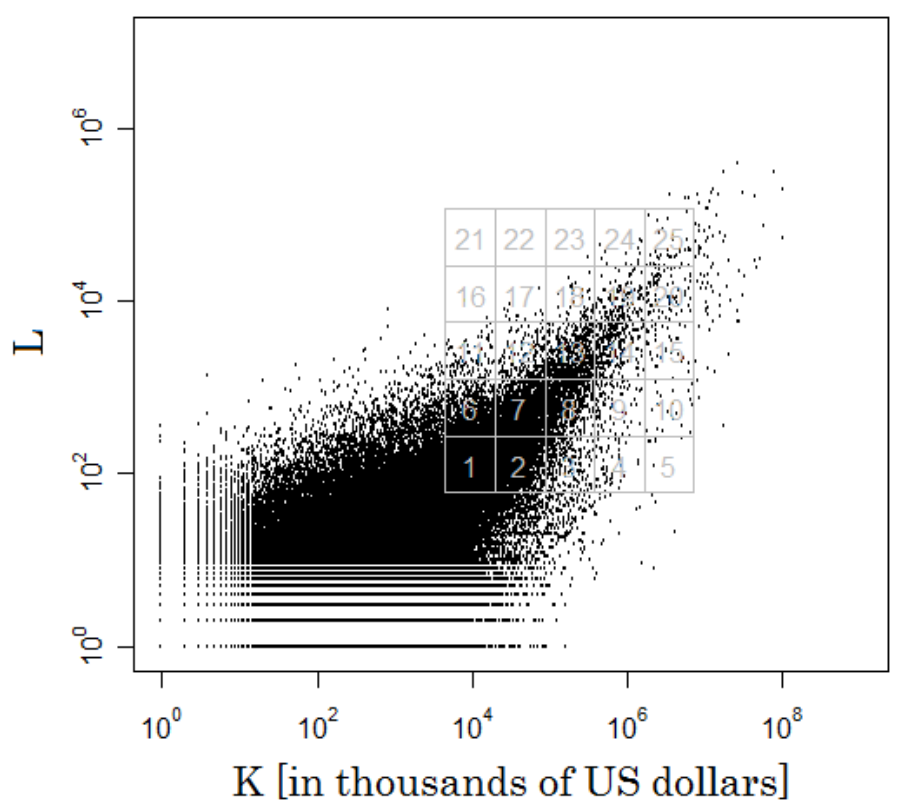

Fig. 1. Scatter plots of tangible fixed assets and the number of workers for Japanese firms in 2008 . We evenly divide the entire $(K, L)$ plane (i.e. $4.5 \times 10^{3} \leq K \leq 7.2 \times 10^{6}$ and $60 \leq L \leq 1.2 \times 10^{5}$ ) into $5 \times 5$ sub-areas.

where

$$
\alpha=\frac{\theta_{1}-\theta_{2}}{\sigma_{\log K}} ; \beta=\frac{\theta_{1}+\theta_{2}}{\sigma_{\log L}} .
$$

Note that, by construction, $Z_{1}$ and $Z_{2}$ are not correlated to each other in the regions $L>L_{0}$ and $K>K_{0}$. Therefore, we can safely apply an ordinary least square to Eq. (2.14) so as to obtain estimates for $\alpha$ and $\beta$.

We run this regression using Japanese data in 2008 to find that $\alpha$ and $\beta$ are equal to 0.18 and 0.85 , respectively. Based on these estimates, we conduct the following exercise. In Figure 1, we plot $K$ and $L$, and divide the entire $(K, L)$ space (i.e. $4.5 \times 10^{3} \leq K \leq 7.2 \times 10^{6}$ and $60 \leq L \leq 1.2 \times 10^{5}$ ) evenly into $5 \times 5$ sub-areas. Then, we produce a probability density function of the estimated TFP for each of the sub-areas. The results are presented in Figure 2. It is confirmed that the probability density functions of $A$ are almost identical irrespective of the values of $K$ and $L$, indicating that the estimated $A$ is indeed independent of $K$ and $L$.

\section{$\S 3$. Estimates of $\alpha$ and $\beta$}

Figure 3 presents the estimates of $\alpha$ and $\beta$ for 30 countries, whose list is available in Table II in the appendix. Figure 3 shows that $\beta$ is greater than $\alpha$ for all countries except Serbia and the Netherlands, suggesting that firm sales depend more closely on the number of workers than on the amount of tangible fixed assets. Figure 4 presents the estimates of $\alpha$ and $\beta$ for different industries. The horizontal axis of Figure 4 represents the SIC (Standard Industrial Classification) code, which is given 


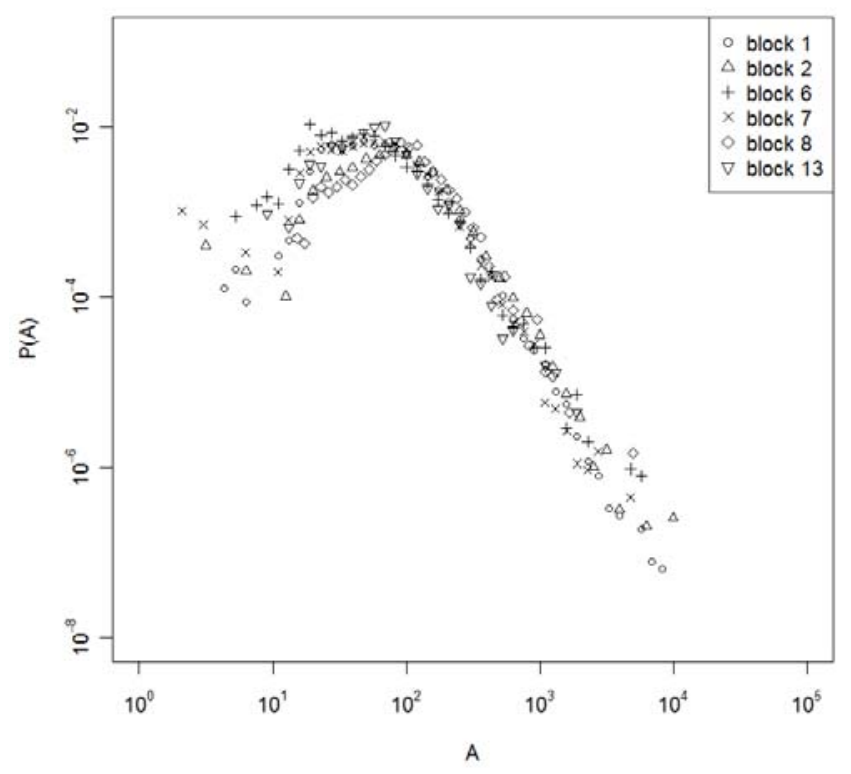

Fig. 2. PDF of total factor productivity. The block number refers to the corresponding sub-area in Figure 1.

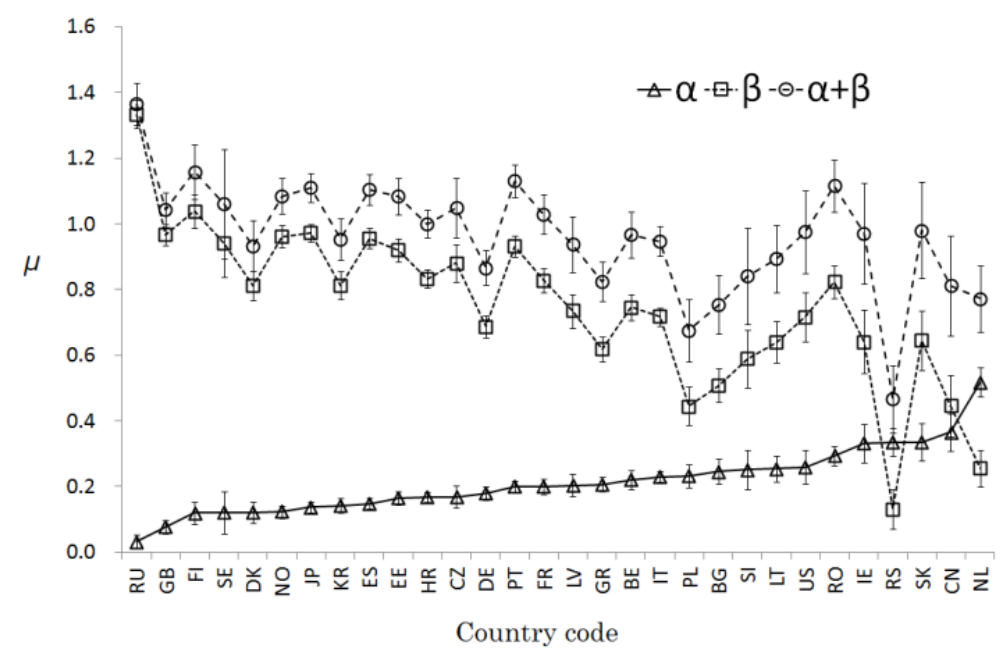

Fig. 3. The estimates of $\alpha, \beta$, and $\alpha+\beta$ for different countries. The horizontal axis represents the country code, which is given in Table II in the appendix.

in Table III in the appendix. ${ }^{22)}$ We see that the estimate of $\alpha$ tends to be somewhere around 0.4 , and $\beta$ tends to be around 0.6 , with the exception of "insurance agents, brokers, and services" (SIC code no.64) where $\beta$ is very close to unity.

Turning to the sum of $\alpha$ and $\beta$, we see in Figure 3 that the sum of $\alpha$ and $\beta$ is close to unity for most countries, although there are several countries, like Serbia, in 


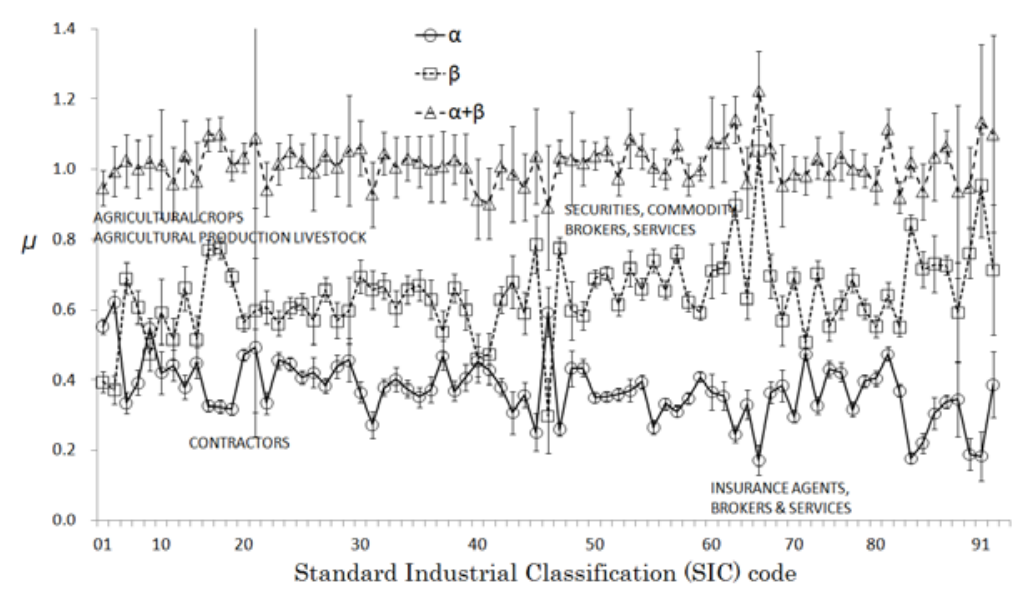

Fig. 4. The estimates of $\alpha, \beta$, and $\alpha+\beta$ for different industries. The horizontal axis represents the SIC code, which is given in Table III in the appendix.

which the sum of $\alpha$ and $\beta$ is significantly smaller than unity. Somewhat interestingly, there is a tendency that the sum of $\alpha$ and $\beta$ is smaller for countries with larger $\alpha$. Also, we see in Figure 4 that the sum of $\alpha$ and $\beta$ is very close to unity for almost all industries. In economics, $\alpha+\beta=1$ implies a constant return to scale; namely, if one increases each of $K$ and $L$ by, say, ten percent, then $S$ also increases by ten percent. The above empirical results suggest that this property holds in most countries, as well as in most industries.

\section{$\S 4$. Total factor productivity}

Given the estimates of $\alpha$ and $\beta$ in hand, we now proceed to the estimation of total factor productivity. Using Eq. (2.10), we calculate total factor productivity as $A=S /\left(K^{\alpha} L^{\beta}\right)$. Figure 5 shows the cumulative distribution functions of total factor productivity obtained in this way for Japanese firms in the years of 2004 to 2008. We see that the tail part of the distribution for each year satisfies:

$$
P_{>}(A) \propto A^{-\mu_{A}} \text { for } A>A_{0} .
$$

indicating that total factor productivity follows a power law distribution with an exponent of $\mu_{A}$. Note that the power law exponent is about 1.65, and it remains unchanged over time.

Figure 6 shows the power law exponents of total factor productivity for 27 countries, together with the power law exponents of sales $\left(\mu_{S}\right)$, the number of workers $\left(\mu_{L}\right)$, and tangible fixed assets $\left(\mu_{K}\right)$. These power law exponents are estimated using a new method proposed by recent studies. ${ }^{9), 23)}$ That is, through a set of statistical tests, we identify a range of a variable in which the variable follows a power law distribution. A power law exponent is estimated using observations only in that range. We see that

$$
\mu_{K}<\mu_{S} \leq \mu_{L}<\mu_{A}
$$




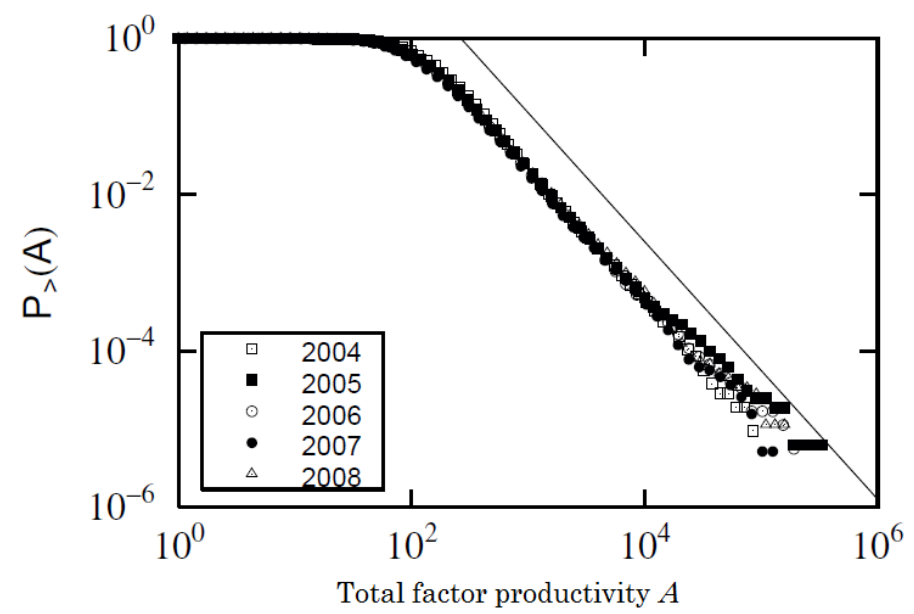

Fig. 5. CDF of total factor productivity for Japanese firms in 2004 to 2008 . The straight reference line represents a power law with an exponent of 1.65 .

Table I. Inequality in terms of productivity, sales, the number of workers, and tangible fixed assets.

\begin{tabular}{lrrrr}
\hline & Productivity & Sales & Number of workers & Tangible fixed assets \\
\hline 20 percent of the total & $1.3 \%$ & $0.019 \%$ & $0.016 \%$ & $0.0067 \%$ \\
50 percent of the total & $23 \%$ & $0.21 \%$ & $0.25 \%$ & $0.066 \%$ \\
80 percent of the total & $43 \%$ & $2.9 \%$ & $5.3 \%$ & $0.90 \%$ \\
\hline
\end{tabular}

holds for almost all countries. This indicates that inequality across firms in terms of $A$ tends to be smaller than inequality in terms of $S, K$, or $L$. To show this result in a different way, we count the number of firms that account for 20,50, 80 percent of the total of $A$. Similarly, we count the number of firms that account for 20,50 , 80 percent of the total of $S, L$, and $K$. The result for Japanese firms in 2008 is presented in Table I, showing that the we need top 23 percent firms to account for 50 percent of the total of $A$, while we need only 0.21 percent firms to account for 50 percent of the total of $S$. Even more surprisingly, we need only 0.066 percent firms to account for 50 percent of the total of $K$.

The result that $\mu_{A}$ is greater than $\mu_{S}$ implies the following. Note that, by construction, $A, Z_{1}$ and $Z_{2}$ in Eq. (2.14) are independent of each other. Therefore, the four power law exponents, $\mu_{S}, \mu_{A}, \mu_{Z_{1}}$, and $\mu_{Z_{2}}$, must satisfy the following: ${ }^{24), 25)}$

$$
\mu_{S}=\min \left\{\mu_{A}, \frac{\mu_{Z_{1}}}{\theta_{1}}, \frac{\mu_{Z_{2}}}{\theta_{2}}\right\} \text {. }
$$

The empirical result that $\mu_{A}$ tends to be greater than $\mu_{S}$ implies that the tail part of the distribution of sales does not stem from the tail part of the distribution of total factor productivity.

Figure 7 shows the power law exponents of total factor productivity for different industries. The three lines represent 2006, 2007, and 2008. We see that $\mu_{A}$ is small 


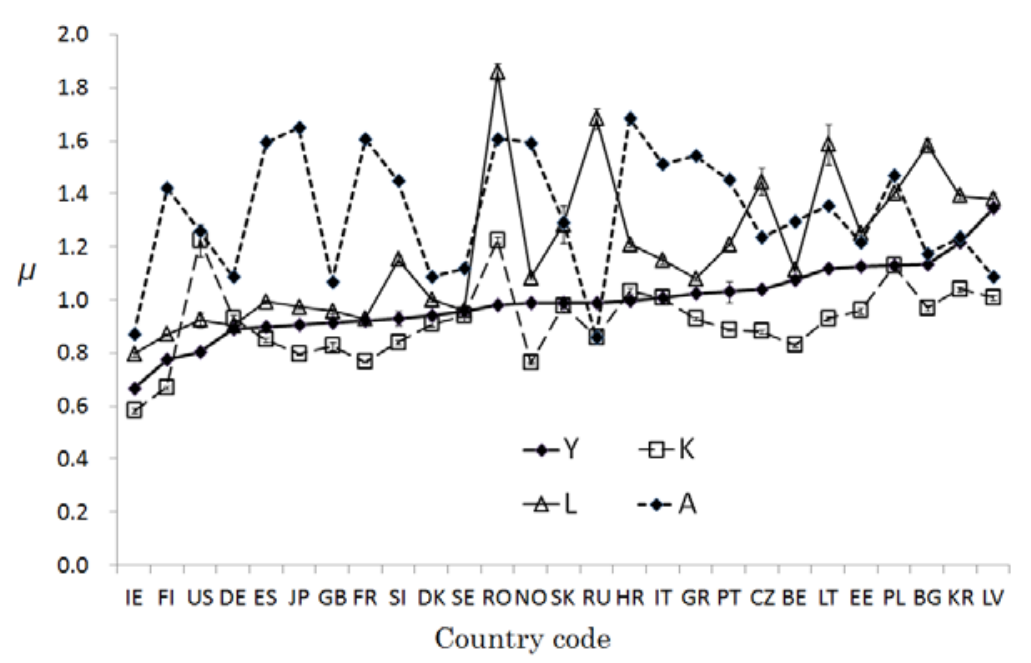

Fig. 6. Power law exponents in 2008 for different countries. The horizontal axis represents the country code, which is given in Table II in the appendix.

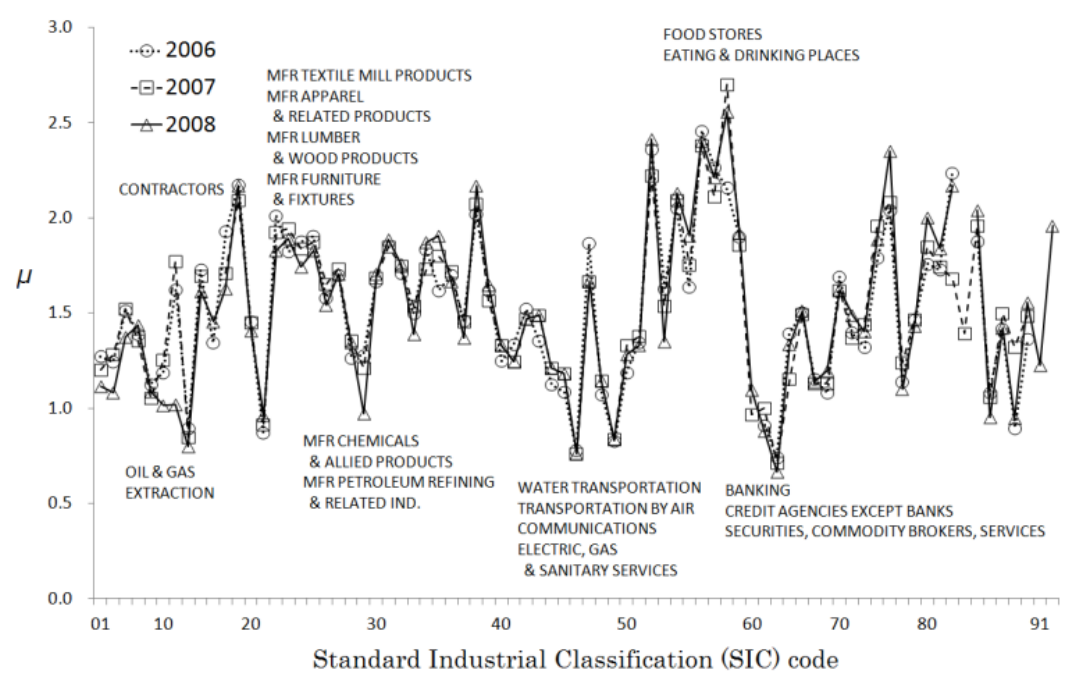

Fig. 7. Power law exponents for different industries. The horizontal axis represents the SIC code, which is given in Table III in the appendix.

for banks, credit agencies, securities, commodity brokers, and services, suggesting that total factor productivity in these industries is significantly unequal across firms. It may be the case that, in these industries, the tail of sales distribution is generated by the tail of productivity distribution. On the other hand, $\mu_{A}$ is relatively large for food stores, restaurants, and automobile repair shops, suggesting that total factor productivity in these industries is not so unequal across firms. It is highly likely that the tail of sales distribution in these industries comes not from the tail of productivity distribution, but from the tail of the number of workers distribution or from the tail of tangible fixed assets distribution. 


\section{$\S 5$. Production function for value added}

So far we have used firm sales as a measure of output. Firm sales data is easy to obtain since it is disclosed by almost all firms in their financial statements, irrespective of which country and which industry a firm belongs to. This is particularly important when one seeks to conduct cross country analysis as we did in the previous sections. However, some economists prefer to use value added as a measure of output, since it may not be appropriate to ignore the role of raw materials and intermediate products in the process of production, at least for some firms belonging to a certain industries. In this section, we check how the results in the previous sections will change when we use value added instead of sales as a measure of output. Unfortunately, value added for each firm is available only for Japanese manufacturing firms. We will use the dataset provided by Teikoku Databank Ltd. for about 21,000 Japanese manufacturing firms in 2008.

Value added and sales are related as:

$$
V=S-F
$$

where $V$ represents value added and $F$ is intermediate inputs, consisting of the expenditures on raw materials, purchased parts, transportations, outsourcing, and so on. We start with looking at the correlations between the value-added to sales ratio, which is defined by $1-F / S$, and $L$ or $K$. The left panel of Figure 8 shows how the distribution of the value-added to sales ratio depends on the value of $L$. Specifically, we divide the range of $L$, which is given by $10 \leq L<10^{3.5}$, evenly into five. For each of the five sub-regions of $L$, we present a probability density function of the value-added to sales ratio conditional on that $L$ belongs to that sub-range. We see that the five PDFs are almost identical, indicating that the value-added to sales ratio is independent of $L$. Similarly, the right panel of Figure 8 shows the conditional distributions of the value-added to sales ratio for different values of $K$. The conditional PDFs are almost identical irrespective of the value of $K$, suggesting again that the value-added to sales ratio is independent of $K$.

Given that the value-added to sales ratio is correlated neither with $L$ nor $K$, we can rewrite Eq. (2.10) as

$$
V=A^{\prime} K^{\alpha} L^{\beta},
$$

where $A^{\prime}$ is defined as $A^{\prime}=(1-F / S) A$. Using Eq. (5.2), we estimate total factor productivity as we did in the previous sections. Figure 9 shows the cumulative distribution function of $A^{\prime}$, together with that of $A$. We confirm that the tail part of the distribution of $A^{\prime}$ is characterized by a power law, and that the power law exponent associated with $A^{\prime}$ is almost identical with the exponent associated with $A$.

\section{$\S 6$. Conclusion}

In this paper we have estimated total factor productivity for mega firms from 30 countries. We found that a productivity distribution is characterized by a power 

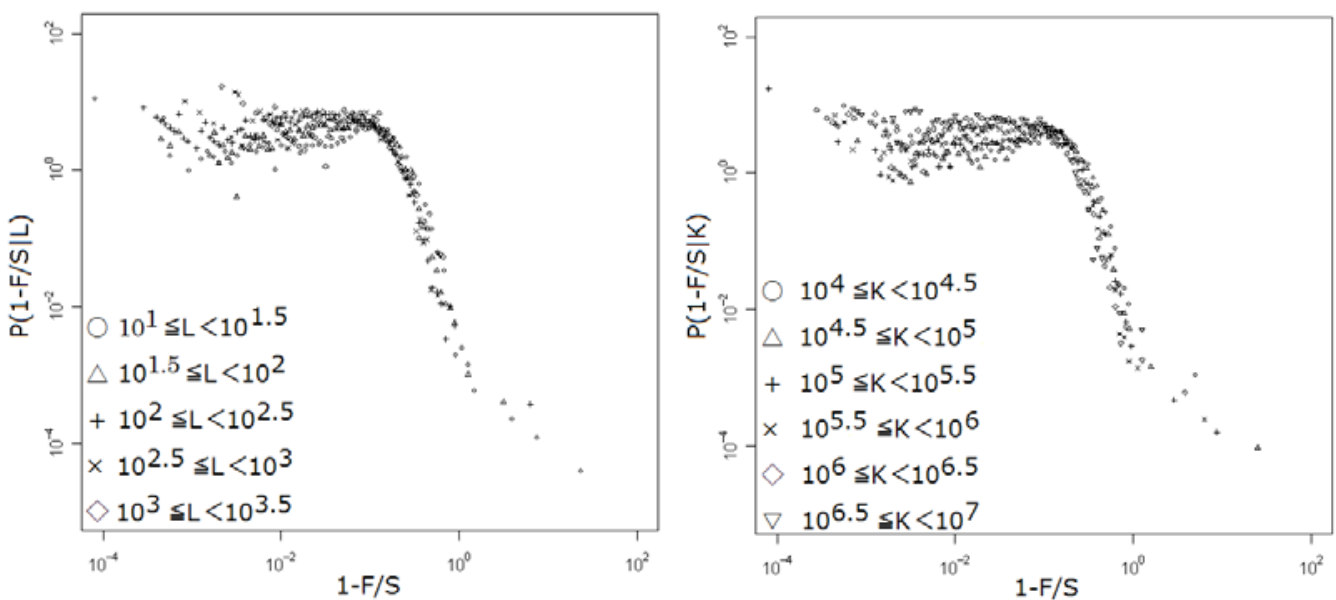

Fig. 8. PDF of the value-added to sales ratio for different values of $L$ (left panel) and for different values of $K$ (right panel). The range of $L, 10 \leq L<10^{3.5}$, is divided evenly into five, while the range of $K, 10^{4} \leq K<10^{7}$, divided evenly into six. The data for Japanese manufacturing firms in 2008 is used.

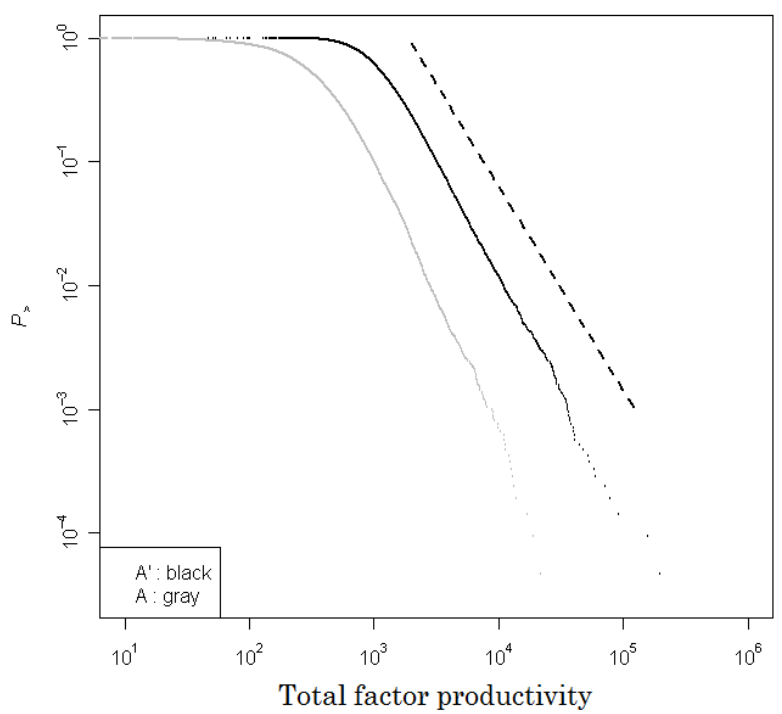

Fig. 9. CDFs of $A$ and $A^{\prime}$ for Japanese manufacturing firms in 2008. The reference line is a power law with an exponent of 1.65 .

law tail, as in the case of other firm size variables, including firm sales, the number of workers, and tangible fixed assets. However, the power law exponent associated with a productivity distribution tends to be greater than those for the other firm size variables. We have confirmed this tendency for different countries, as well as for different industries. This result suggests that the tail part of a sales distribution does not stem from the tail of a productivity distribution, but from the tail of the distributions of the number of workers or tangible fixed assets. 


\section{Acknowledgements}

The authors thank the Yukawa Institute for Theoretical Physics at Kyoto University, where this work was completed during the YITP-W-11-04 on "Econophysics 2011". This work was supported in part by a Grant-in-Aid for Scientific Research (C) (No. 20510147) from the Ministry of Education, Culture, Sports, Science and Technology, Japan.

\section{References}

1) V. Pareto, Cours d'Economique Politique (Macmillan, London, 1897).

2) H. A. Simon and C. P. Bonini, The American Economic Review 48 (1958), 67-617.

3) R. E. Quandt, The American Economic Review 56 (1966), 416-432.

4) Y. Ijiri and H. A. Simon, Journal of Political Economy 82 (1974), 315-331.

5) K. Okuyama, M. Takayasu, and H. Takayasu, Physica A 269 (1999), 125.

6) M. H. R. Stanley, S. V. Buldyrev, S. Havlin, R. Mantegna, M.A. Salinger, and H. E. Stanley, Economics Letters 49 (1995), 453-457.

7) T. Mizuno, M. Katori, H. Takayasu, and M. Takayasu, Empirical Science of Financial Fluctuations - The Advent of Econophysics (Springer Verlag, Tokyo, 2002) 321.

8) R. L. Axtell, Science 293 (2001), 1818.

9) S. Fujimoto, A. Ishikawa, T. Mizuno, and T. Watanabe, submitted to Economics.

10) R. N. Mantegna and H. E. Stanley, Nature 376 (1995), 46-49.

11) T. Mizuno, M. Takayasu, and H. Takayasu, Physica A 332 (2004), 403-411.

12) A. Ishikawa, S. Fujimoto, and T. Mizuno, Physica A 390 (2011), 4273-4285.

13) Y. Fujiwara, C. D. Guilmi, H. Aoyama, M. Gallegati, and W. Souma, Physica A 335 (2004), 197-216.

14) A. Ishikawa, S. Fujimoto, T. Watanabe, and T. Mizuno, submitted to EPJ B.

15) Y. Ikeda and W. Souma, Progress of Theoretical Physics Supplement 179 (2009), 93.

16) H. Aoyama, H. Yoshikawa, H. Iyetomi, and Y. Fujiwara, Progress of Theoretical Physics Supplement 179 (2009), 80.

17) T. Watanabe, T. Mizuno, A. Ishikawa, and S. Fujimoto, The Economic Review 62 (2011), 193-208.

18) H. S. Houthakker, The Review of Economic Studied 23 (1955), 27-31.

19) S. Rosen, Economica 45 (1978), 235-250.

20) C. I. Jones, Quarterly Journal of Economics 120 (2005), 517-549.

21) C. W. Cobb and P. H. Douglass, The American Economic Review 18 (1928), 139-165.

22) U.S. Department of Labor, http://www.dol.gov/

23) Y. Malevergne, V. Pisarenko, and D. Sornette, Physical Review E 83 (2011), 036111.

24) A. H. Jessen and T. Mikosch, Publ. de l'Inst. Math. 94 (2006), 171-192.

25) X. Gabaix, Annual Review of Economics 1 (2009), 255-294. 


\section{Appendix}

Table II. Country code

\begin{tabular}{llllll}
\hline \hline BE & Belgium & BG & Bulgaria & CN & China \\
CZ & Czech & DE & Germany & DK & Denmark \\
EE & Estonia & ES & Spain & FI & Finland \\
FR & France & GB & The U.K. & GR & Greece \\
HR & Croatia & IE & Ireland & IT & Italy \\
JP & Japan & KR & Korea & LT & Lithuania \\
LV & Latvia & NL & The Netherlands & NO & Norway \\
PL & Poland & PT & Portugal & RO & Romania \\
RS & Serbia & RU & Russia & SE & Sweden \\
SI & Slovenia & SK & Slovak & US & United States \\
\hline \hline
\end{tabular}

Table III. Standard Industrial Classification (SIC) code

\begin{tabular}{ll}
\hline \hline $01-09$ & Forestry and Fishery \\
10-19 & Mining, Building, Construction \\
$20-29$ & Food, Textile, Wood, Chemicals \\
$30-39$ & Rubber, Leather, Metal, Machinery \\
$40-49$ & Railroad, Transportation, Postal, Telecom, Electricity, Gas, Water Supply \\
$50-59$ & Wholesale, Retailing, Eatery \\
$60-69$ & Bank, Finance, Security, Insurance, Real estate, Investor \\
$70-79$ & Services \\
$80-89$ & Medical, Legal, Educational, Social Services \\
$90-99$ & International Affairs and Non-Operating Establishments \\
\hline \hline
\end{tabular}

